\section{NSCLC: Therapie mit oder ohne Platin?}

Die meisten Patienten mit einem nichtkleinzelligen Lungenkarzinom (NSCLC) werden mit einer Platindoppeltherapie behandelt. Um die Therapie verbessern zu können, wäre ein prognostischer Marker hilfreich.

n retrospektiven Studien war die Expression des Proteins ERCC1 (Excision Repair Cross Complementation Group 1) beim NSCLC mit einer Platinresistenz und dem Überleben assoziiert. Nun wurde es erstmals in einer prospektiven, randomisierten Studie als prädiktiver und prognostischer Biomarker untersucht. Ziel war es, die Überlegenheit einer nicht platinbasierten Chemotherapie über eine Platindoublette bei
ERCC1-positivem NSCLC zu zeigen und die Nichtunterlegenheit bei ERCC1Negativität.

Chemotherapienaive Patienten mit NSCLC (Stadium IIIB und IV) und Plattenepithelhistologie $(\mathrm{n}=177 ; 76,7 \%$ ERCC1-positiv, $68,5 \%$ positiv für den ERCC1-Liganden XPF) erhielten randomisiert Cisplatin/Gemcitabin oder Paclitaxel/Gemcitabin. Patienten mit Nichtplattenepithelhistologie $(n=471 ; 54,5 \%$
Tab. 1: Gesamtüberleben bei Patienten mit Nichtplattenepithelhistologie und ERCC1-Positivität

\begin{tabular}{|l|l|l|l|}
\hline & Zahl der Patienten (Ereignisse) & HR (95\%-KI); & p-Wert \\
\hline Cisplatin + Pemetrexed & $230(198)$ & $1,05(0,80-1,40)$ & 0,72 \\
\hline Paclitaxel + Pemetrexed & $234(201)$ & $1,14(0,86-1,51)$ & 0,36 \\
\hline Kombiniert* & $464(399)$ & $1,11(0,91-1,35)$ & 0,32 \\
\hline
\end{tabular}

$H R=$ Hazard Ratio; $95 \%-K I=95 \%$-Konfidenzintervall; *HR adjustiert für die Therapiegruppe
ERCC1-positiv, 70,5\% XPF-positiv) bekamen Cisplatin/Pemetrexed oder Paclitaxel/Pemetrexed. Primärer Endpunkt war das Gesamtüberleben.

Die Rekrutierung von Patienten wurde frühzeitig beendet: bei Patienten mit Plattenepithelkarzinom im Jahr 2012, weil das Gesamtüberleben unter der Therapie ohne Platin kürzer war (median 7,6 vs. 10,7 Monate; Hazard Ratio [HR] 1,46; $\mathrm{p}=0,02)$; bei Patienten mit Nichtplattenepithelkarzinom 2013. In dieser Gruppe war das mediane Gesamtüberleben mit und ohne Platin vergleichbar (bei ERCC1-positiven Patienten 8,0 Monate unter Paclitaxel/Pemetrexed vs. 9,6 Monate unter Cisplatin/Pemetrexed; HR 1,11, Tab. 1; bei ERCC1-negativen Patienten median 10,3 vs. 11,6 Monate; HR $0,99 ; \mathrm{p}=0,64)$.

Fazit: Beim Plattenepithelkarzinom war die Platintherapie der Chemotherapie ohne Platin überlegen. Eine Therapiesteuerung durch ERCC1 oder XPF ist nicht sinnvoll.

Judith Neumaier

Lee SM et al. Randomized Prospective Biomarker Trial of ERCC1 for Comparing Platinum and Nonplatinum Therapy in Advanced Non-SmallCell Lung Cancer: ERCC1 Trial (ET). J Clin Oncol. 2017;35(4):402-11.

zard Ratio [HR] 1,27; $p=0,067)$, das mediane progressionsfreie Überleben lag bei 2,7 versus 2,6 Monaten (HR 0,99; $\mathrm{p}=0,92)$. Die Gesamtansprechrate war ebenso vergleichbar (8,4 vs. 9,6\%).

Auch nach weiteren Untersuchungen des MET-Status ergab sich kein Nutzen für Onartuzumab. Patienten mit EGFRMutationen zeigten unter Onartuzumab einen Trend zu kürzerem Überleben (HR 4,68, 95\%-Konfidenzintervall 0,97-22,63).

Fazit: Die Therapie mit Onartuzumab plus Erlotinib konnte die Prognose des MET-positiven, fortgeschrittenen NSCLC nicht verbessern. Auch bei anderen Tumoren führte dieser Antikörper bisher nicht zum Erfolg. Judith Neumaier

Spigel DR et al. Results From the Phase III Randomized Trial of Onartuzumab Plus Erlotinib Versus Erlotinib in Previously Treated Stage IIIB or IV Non-Small-Cell Lung Cancer: MET Lung. J Clin Oncol. 2017;35(4):412-20. 